\title{
Impact of Molecular Orientation and Packing Density on Electronic Polarization in the Bulk and at Surfaces of Organic Semiconductors
}

Sean M Ryno, Chad Risko, and Jean-Luc Bredas

ACS Appl. Mater. Interfaces, Just Accepted Manuscript • DOI: 10.1021/acsami.6b02579 • Publication Date (Web): 16 May 2016

Downloaded from http://pubs.acs.org on May 22, 2016

\section{Just Accepted}

"Just Accepted" manuscripts have been peer-reviewed and accepted for publication. They are posted online prior to technical editing, formatting for publication and author proofing. The American Chemical Society provides "Just Accepted" as a free service to the research community to expedite the dissemination of scientific material as soon as possible after acceptance. "Just Accepted" manuscripts appear in full in PDF format accompanied by an HTML abstract. "Just Accepted" manuscripts have been fully peer reviewed, but should not be considered the official version of record. They are accessible to all readers and citable by the Digital Object Identifier (DOI®). "Just Accepted" is an optional service offered to authors. Therefore, the "Just Accepted" Web site may not include all articles that will be published in the journal. After a manuscript is technically edited and formatted, it will be removed from the "Just Accepted" Web site and published as an ASAP article. Note that technical editing may introduce minor changes to the manuscript text and/or graphics which could affect content, and all legal disclaimers and ethical guidelines that apply to the journal pertain. ACS cannot be held responsible for errors or consequences arising from the use of information contained in these "Just Accepted" manuscripts. 


\title{
Impact of Molecular Orientation and Packing Density on
}

\author{
Electronic Polarization in the Bulk and at Surfaces
}

\author{
of Organic Semiconductors
}

\author{
Sean M. Ryno, ${ }^{\ddagger, \|}$ Chad Risko, ${ }^{\#, *}$ and Jean-Luc Brédas ${ }^{\ddagger, *}$ \\ ${ }^{\ddagger}$ Solar and Photovoltaics Engineering Research Center \\ Division of Physical Science and Engineering \\ King Abdullah University of Science and Technology \\ Thuwal 23599-6900, Kingdom of Saudi Arabia
}

"School of Chemistry and Biochemistry \& Center for Organic Photonics and Electronics Georgia Institute of Technology Atlanta, Georgia 30332-0400

\author{
\# Department of Chemistry \\ \& Center for Applied Energy Research \\ University of Kentucky \\ Lexington, Kentucky 40506-0055
}

\begin{abstract}
*Corresponding authors: chad.risko@kyu.edu; jean-luc.bredas@,kaust.edu.sa
\end{abstract}
Keywords: Intermolecular interactions, organic semiconductors, polarization energy, bulk, surface, molecular packing 


\begin{abstract}
The polarizable environment surrounding charge carriers in organic semiconductors impacts the efficiency of the charge transport process. Here, we consider two representative organic semiconductors, tetracene and rubrene, and evaluate their polarization energies in the bulk and at the organic-vacuum interface using a polarizable force field that accounts for induced-dipole and quadrupole interactions. Though both oligoacenes pack in a herringbone motif, the tetraphenyl substituents on the tetracene backbone of rubrene alter greatly the nature of the packing. The resulting change in relative orientations of neighboring molecules is found to reduce the bulk polarization energy of holes in rubrene by some $0.3 \mathrm{eV}$ when compared to tetracene. The consideration of model organic-vacuum interfaces highlights the significant variation in the electrostatic environment for a charge carrier at a surface although the net change in polarization energy is small; interestingly, the environment of a charge even just one layer removed from the surface can be viewed already as representative of the bulk. Overall, it is found that in these herringbone-type layered crystals the polarization energy has a much stronger dependence on the intralayer packing density than interlayer packing density.
\end{abstract}

Keywords: organic semiconductors, polarization energy, molecular packing, organic interfaces 


\section{Introduction}

Organic electronic materials such as the oligoacenes, either unsubstituted (i.e., naphthalene, tetracene, and pentacene) or substituted (i.e., rubrene and TIPS-pentacene), have drawn the attention of materials chemists for years because of the potential to modulate their chemical structure to induce better charge-carrier transport properties. ${ }^{1-12}$ Charge transport in these materials is dependent on the strength of the electronic couplings between adjacent molecules, the extent of geometric and lattice relaxations upon charge injection (ionization), and the differences in site energies. Each of these factors can be affected by changes in chemical structure, processing conditions, and the presence of defects. In addition, in particular the site energies are influenced by a polarizable environment in the solid state. ${ }^{13-15}$

Polarization effects are quantified by the associated solid-state polarization energy, typically defined by the Lyons model. ${ }^{14,16}$ For a positive charge, the polarization energy $\left(P_{+}\right)$is defined as the difference between the gas-phase $\left(I E_{g}\right)$ and solid-state $\left(I E_{S S}\right)$ ionization energies, and generally falls between 1.5 to $2.0 \mathrm{eV}$ for $\pi$-conjugated organic materials, ${ }^{14,17-18}$ a similar relationship exists for a negative charge, based on the electron affinities. An important feature to recall regarding the polarization process is that its electronic and nuclear components occur on different time scales: $10^{-16}-10^{-15} \mathrm{~s}$ for electrons and $10^{-14}-10^{-11} \mathrm{~s}$ for nuclei. Hence, these two contributions can be separated (in a way analogous with the Born-Oppenheimer approximation) to allow the assessment of the electronic polarization energy component on the basis of a frozen molecular geometry. ${ }^{14-15}$

We previously demonstrated that, even though pentacene and TIPS-pentacene [6,13bis(triisopropylsilylethynyl)pentacene] are electronically similar, the differences in their molecular packings (the TIPS groups translating the preferred herringbone packing motif of 
pentacene to a two-dimensional brickwork configuration) have a significant effect on the hole polarization energies. In fact, the hole polarization energy of pentacene is larger by up to a few tenths of an $\mathrm{eV}{ }^{19}$ These results also underlined that great care must be taken when extrapolating data that neglect the variations in packing configurations, e.g., the use of cyclic voltammetry data in solution, to predict solid-state ionization energies.

Using the polarizable force-field methodology previously reported, ${ }^{19-20}$ we investigate here the polarization effects in two other representative organic semiconductors, tetracene and rubrene (5,6,11,12-tetraphenyltetracene). These two molecular systems share the same conjugated backbone; however, while they both pack in a herringbone fashion (Figure 1), the presence of the four phenyl groups in rubrene alters the nature of the herringbone packing configuration. In rubrene, the short-axis (head) of one tetracene interacts with the $\pi$-plane of the nearest herringbone neighbor, while in unsubstituted tetracene, it is the long-axis edge of one molecule that interacts with the $\pi$-plane of its herringbone neighbor (as is usually the case in unsubstituted oligoacenes), see Figures $1 \mathrm{~b}$ and $1 \mathrm{f}$. We also explore the effect of changing the packing density on the polarization energy, and contrast the dependence on the packing in the $a b$-plane, that is within the molecular layer (Figures $1 \mathrm{~b}$ and $1 \mathrm{f}$ ), with respect to that along the $c$-axis, that is between molecular layers (Figures $1 \mathrm{c}$ and $1 \mathrm{~g}$ ). We note that along the $c$-axis, interlayer interactions are dominated by peripheral hydrogen contributions, while within the $a b$-plane there are significant $\pi$-conjugated backbone interactions.

In addition to the effects of molecular orientation and packing density of the material, we evaluate the differences between the polarization energy in the bulk versus at the organicvacuum interface. In 1978, Salaneck measured a $0.3 \mathrm{eV}$ smaller ionization energy for an anthracene molecule on the very surface with respect to a molecule just under the surface. ${ }^{21}$ In 
recent years, this result has garnered additional interest with various experimental and theoretical reports examining the differences in ionization and polarization energies. ${ }^{22-25}$ Here, by using a model that allows for the separation of individual interactions, we are able to rationalize the lower polarization energy at the organic-vacuum interface by decomposing the polarization into static interactions (that is, the charge-permanent multipole interactions) and dynamic interactions (charge-induced dipole interactions). ${ }^{20}$ Though the resulting change in observed IE at a surface may be small, the energetics at the surface can be tuned by controlling separately the static and dynamic interactions. We also examine the polarization energy at the organic-vacuum interface as a function of charge carrier depth within the organic material. Our focus will be on the polarization energies associated with the presence of an excess positive charge, since tetracene and rubrene are well established hole transporters. 
a)

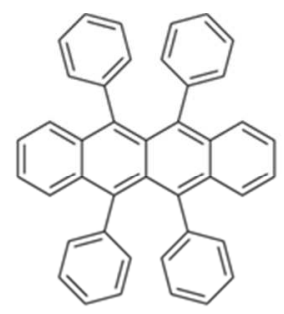

b)

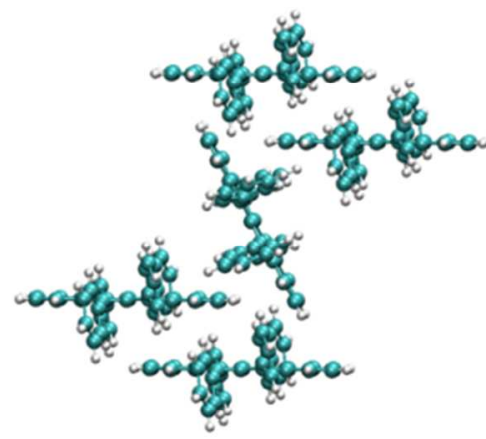

c)

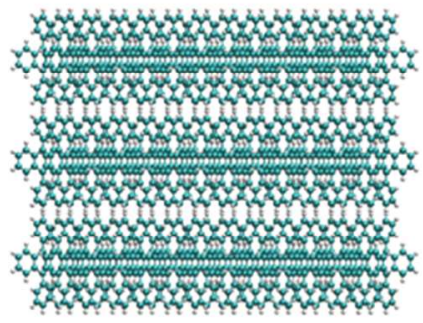

d)

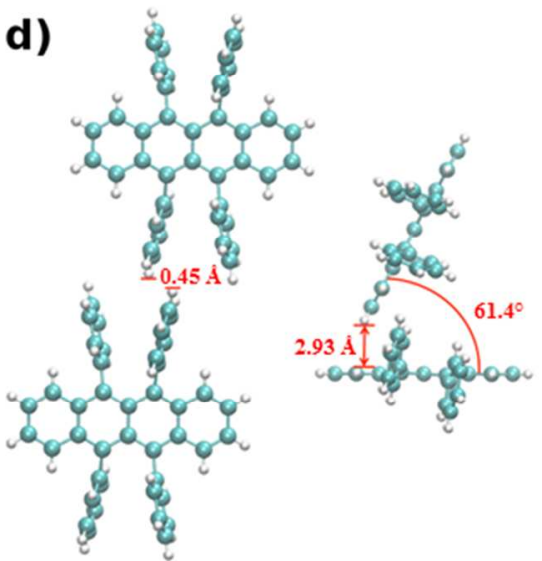

e)

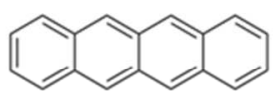

f)
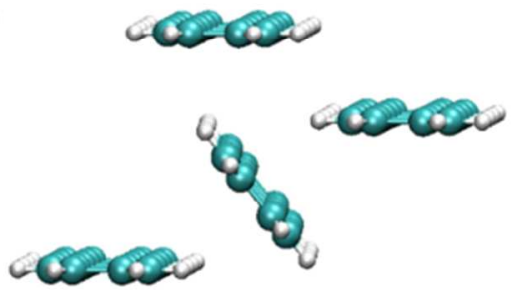

g)

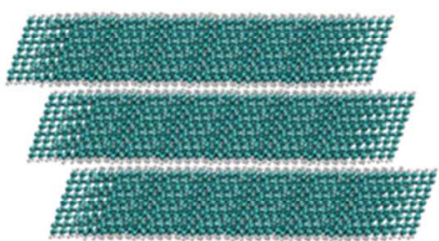

h)

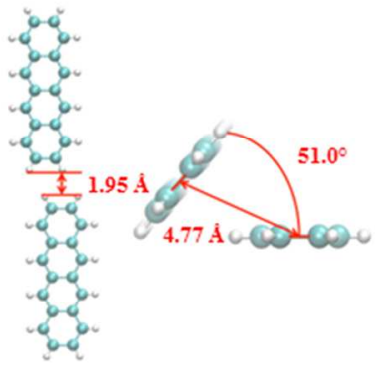

Figure 1. (a and e) Chemical structures of rubrene and tetracene, respectively. (b and f) Intralayer ( $a / b$-plane) packing in rubrene and tetracene. Both systems present herringbone packing, with rubrene displaying as well significant backbone overlap. (c and g) Interlayer (caxis) packing in rubrene and tetracene. ( $\mathrm{d}$ and $\mathrm{h}$ ) Illustration of the intermolecular distances and angles between layers, and dimer separation and angles within the layers. ${ }^{26-27}$ 


\section{Computational Methodology}

The geometries for all molecules used in this study were taken from the crystal structures for tetracene $\left(\right.$ TETCEN01) ${ }^{26}$ and rubrene (QQQCIG04) ${ }^{27}$ deposited in the Cambridge Structural Database (CSD; CSD identification codes are given within parentheses). ${ }^{28-29}$ Supercells, constructed from the experimental unit cells, were used to generate spherical clusters, cylinders, and slabs for the polarization energy studies. Modified unit cells were considered to explore the effect of packing density $\left(1.44 \mathrm{~g} / \mathrm{cm}^{3}\right.$, tetracene; $1.30 \mathrm{~g} / \mathrm{cm}^{3}$, rubrene $){ }^{30}$ they were constructed by increasing the unit-cell parameters while leaving the internal coordinates of the molecules unchanged, thus increasing the interlayer spacing, intralayer spacing, or both. Spherical clusters [cylinders] were constructed where molecules with their center-of-mass inside a given radius [and height] are selected from a larger supercell. The molecule carrying the excess charge in the polarization energy calculations is defined as the origin in all systems. Organic-vacuum interfaces were created by defining the origin of the system at the molecule in the center of the layer with the largest Z-axis value (i.e., the top-most layer of the supercell). A cylinder was then extracted in the same manner as above, but where half of the cylinder is composed of vacuum. Here, ionization is taken to occur via a vertical process; thus, the neutral crystal geometry is used for the charged systems.

All calculations of bulk polarization energy were carried out with our previously described methodology ${ }^{20}$ using the AMOEBA force field of Ponder and co-workers. ${ }^{31-32}$ The electrostatic parameters were derived from a distributed multipole analysis (DMA). Single-particle density matrices for the neutral, radical-cation, and radical-anion states for the DMA calculations were determined via single-point energy calculations using second-order Møller-Plesset perturbation theory (MP2) and a 6-31+G(d,p) basis; the MP2 calculations were carried out with the Gaussian 
09 (revisions A.02 and B.01) code. $^{33-34}$ Gas-phase and solid-state ionization energies and electron affinities were determined through the AMOEBA-based calculations as previously described. $^{20}$ These energies were then input into the Lyons model:

$$
\begin{aligned}
& P_{+}=I E_{s s}-I E_{g} \\
& P_{-}=E A_{s s}-E A_{g}
\end{aligned}
$$

where $P_{+}\left[P_{-}\right]$is the polarization energy due to a positive [negative] charge and $I E[E A]$ is the solid-state or gas-phase ionization energy [electron affinity], respectively, to determine the polarization energy of a particular cluster. Bulk polarization energies were determined by increasing the system size and plotting the polarization energy versus the cube root of the number of molecules in the spherical clusters. For cylindrical systems, the polarization energy was plotted versus the square root of the average number of molecules per layer to remove the height dependence of the systems.

\section{Results and Discussion}

\section{a) Impact of Molecular Orientations}

While the packings of rubrene and tetracene are both of herringbone type, there exist critical differences: Tetracene displays only (long-axis) edge-to-face packing (Figure 1f), while rubrene displays both (short-axis) edge-to-face and face-to-face interactions (Figure 1b). As we previously reported, ${ }^{19}$ the differences in the packing configurations of pentacene and TIPSpentacene strongly impact the polarization energy, since the variations in electrostatic interactions influence the amount of stabilization experienced by an excess charge. While the differences in packing between tetracene and rubrene are not as overtly obvious as those between pentacene and TIPS-pentacene, they are nevertheless expected to impact the polarization energy. 
In fact, the polarization energies of rubrene and tetracene were initially reported to differ by some $0.7 \mathrm{eV}$ as Sato and co-workers measured a polarization energy of $1.1 \mathrm{eV}$ for a positive charge carrier in rubrene and $1.8 \mathrm{eV}$ in tetracene. ${ }^{17}$ Note that a later re-evaluation reported the polarization energy of tetracene to be $1.6 \mathrm{eV},{ }^{18}$ in agreement with the early measurements of Pope et $a l .^{9}$ and the theoretical results of Berry et al.; ${ }^{18,35}$ to the best of our knowledge, no additional studies of the polarization energy of rubrene have been reported.

Overall, to provide a baseline for comparison that will be used to better assess the impact that the different packing motifs have on the polarization energies, we first determine the bulk polarization energies of tetracene and rubrene. We find the polarization energy due to a positive charge in tetracene $(1.04 \mathrm{eV})$ to be $0.3 \mathrm{eV}$ larger than that of rubrene $(0.76 \mathrm{eV})$, which is in line with the difference of $0.5 \mathrm{eV}$ between the re-evaluated polarization energy of tetracene and the initial report of rubrene, see Figure S1. The polarization energies for a negative charge are 0.92 $\mathrm{eV}$ and $0.65 \mathrm{eV}$ for rubrene and tetracene, respectively.

To determine where this difference in polarization energy originates, we consider a stepwise approach. First, we examine the permanent multipole interactions among neutral molecules in simple dimer models extracted from the crystal structure. As the quadrupole moments are the largest non-zero multipole moments in rubrene and tetracene, we use the Distributed Multipole Analysis (DMA) ${ }^{36}$ to calculate the quadrupolar electrostatic interactions in dimers of rubrene and tetracene; this allows us to determine the electrostatic effect of changing the orientations of the molecular backbones and altering the herringbone packing. In tetracene, due to the stabilizing edge-to-face interactions, the quadrupole-quadrupole interactions are stabilizing by about 0.04 $\mathrm{eV}$, while in rubrene these stabilizing interactions are twice as large, $0.10 \mathrm{eV}$. Because of the fashion in which rubrene packs, there is an additional dimer configuration that must be 
considered, the face-to-face dimer. In this configuration, the quadrupole interactions are destabilizing by $0.26 \mathrm{eV}$, a value identical to that found for the $\pi$-stacked backbones in TIPSpentacene. Thus, we observe stabilizing permanent quadrupole-quadrupole interactions in neutral tetracene and a mixture of stabilizing and destabilizing interactions in rubrene due to the differences in packing.

Next, to assess how these intrinsic differences in electrostatic interactions extend to the very large cluster systems used for the polarization energy calculations, we consider finite-size clusters of 4-nm radius and compare the electrostatic interactions in these large systems as the charge of the cluster is changed from 0 to \pm 1 . In the absence of induced dipoles, with respect to the neutral systems, the static multipole interactions for a tetracene anion are stabilizing by 0.30 $\mathrm{eV}$, while a rubrene anion is destabilized by $0.22 \mathrm{eV}$. While the excess charge localized on the central molecule of the cluster is distributed across the face of the backbone in both systems, this difference is a direct result of the variations in molecular orientations in the tetracene and rubrene crystals. In tetracene, the excess charge results in each carbon possessing a larger partial negative charge that interacts with a positive quadrupole component on neighboring molecules, while the peripheral hydrogens still possess a small partial positive charge and interact with a negative quadrupole component on neighboring molecules; this results in an overall stabilization due to static multipole interactions. In rubrene, the dominant interaction is the face-to-face interaction that becomes more repulsive and destabilizing. For the cationic species, again in the absence of induced dipoles, there would occur destabilizations with respect to the neutral systems by $1.16 \mathrm{eV}$ and $1.49 \mathrm{eV}$ for tetracene and rubrene, respectively. This is due to the faceto-edge interactions in tetracene becoming essentially positive/positive interactions; the same holds true in rubrene. The rubrene face-to-face interactions remain destabilizing as the carbons of 
the backbone possess partial negative charge. Importantly, when the induced dipoles are turned on, each system experiences an overall stabilization in the range of $0.5 \mathrm{eV}-1.1 \mathrm{eV}$, as induced dipoles always act as a strong stabilizing force.

Lastly, since the induced dipoles can be calculated separately from the static multipole interactions, we break down the polarization energy experienced by an excess charge into:

(i) a static component: $S_{ \pm}=S_{N}-S_{h}$, where $S_{N}$ and $S_{h}$ denote the change in static interactions (thus, taking into account only the permanent higher-order multipoles) in going from single molecules to clusters that are neutral $\left(S_{N}\right)$ or possess a \pm 1 charge $\left(S_{h}\right)$; and

(ii) a dynamic component: $D_{ \pm}=D_{N}-D_{h}$, where $D_{N}$ and $D_{h}$ denote the change in induced interactions in going from single molecules to clusters that are neutral $\left(D_{N}\right)$ or possess a \pm 1 charge $\left(D_{h}\right)$; here, the interactions between permanent multipoles and induced dipoles and the interactions among induced dipoles are considered.

The polarization energy can therefore be defined as the sum of the stabilization of a system due to static and dynamic contributions with respect to a neutral system: $P_{ \pm}=S_{ \pm}+D_{ \pm}$. In the case of an excess positive charge, the static component of the polarization energy in 4-nm finite-size clusters, $S_{+}$, is evaluated to be stabilizing by $0.18 \mathrm{eV}$ in tetracene and by $0.15 \mathrm{eV}$ in rubrene; thus, in both cases, the static electrostatic interactions change by approximately the same amount on going from a neutral system to a positively charged system. When the dynamic component of the polarization energy is considered, the tetracene and rubrene cations are stabilized by $0.74 \mathrm{eV}$ and $0.50 \mathrm{eV}$, respectively, see Table 1 . Adding together the difference between the static and dynamic components of rubrene and tetracene gives a value of $0.27 \mathrm{eV}$, which almost entirely 
accounts for the difference of $0.28 \mathrm{eV}$ between the bulk of the materials (the extrapolation from these finite-size clusters to the bulk thus adds very little). For both tetracene and rubrene, the dynamic contributions are found to be some three times more stabilizing than the static contributions, which highlights the prevalent role of the dominant dynamic interactions in the polarization energy.

It is also useful to explore cylindrical systems. Indeed, while spherical clusters allow us to calculate the bulk polarization energy of a material, cylindrical systems allow for the determination of the polarization energy as a function of the average number of molecules per layer; in this way, a direct evaluation of the impact of neighboring layers can be obtained. Since the cylindrical systems do not have the $1 / N^{3}$ dependence of the spherical systems, the polarization energy has instead to be plotted versus $1 /(N / L)^{2}$, where $\mathrm{N}$ is the number of molecules in the system and $\mathrm{L}$ is the number of layers in the cylinder. For these cylindrical systems, an initial disk (consisting of just one layer) is expanded by symmetrically increasing the number of layers on either side of the disk. Figures 2 and 3 show the polarization energies for rubrene and tetracene cylinders consisting of $1,3,5$, and 7 layers. When the size of the cylinder increases, the polarization energy per layer increases, but the magnitude of the increase quickly diminishes after the nearest-neighbor layers are added. From Figure 2, it can be seen that the $P_{+}$ polarization energy for a single disk of tetracene is $0.84 \mathrm{eV}$ and increases by $0.16 \mathrm{eV}$ when the nearest-neighbor layers are added. The polarization energy then only increases by an additional $0.02 \mathrm{eV}$ for the next nearest-neighbor layers. The large impact of the nearest-neighbor layers highlights the importance of their inclusion in understanding the ionization energies of organic molecular materials; clearly, as we discuss below, this feature has significant impact when examining the polarization energy at surfaces. 

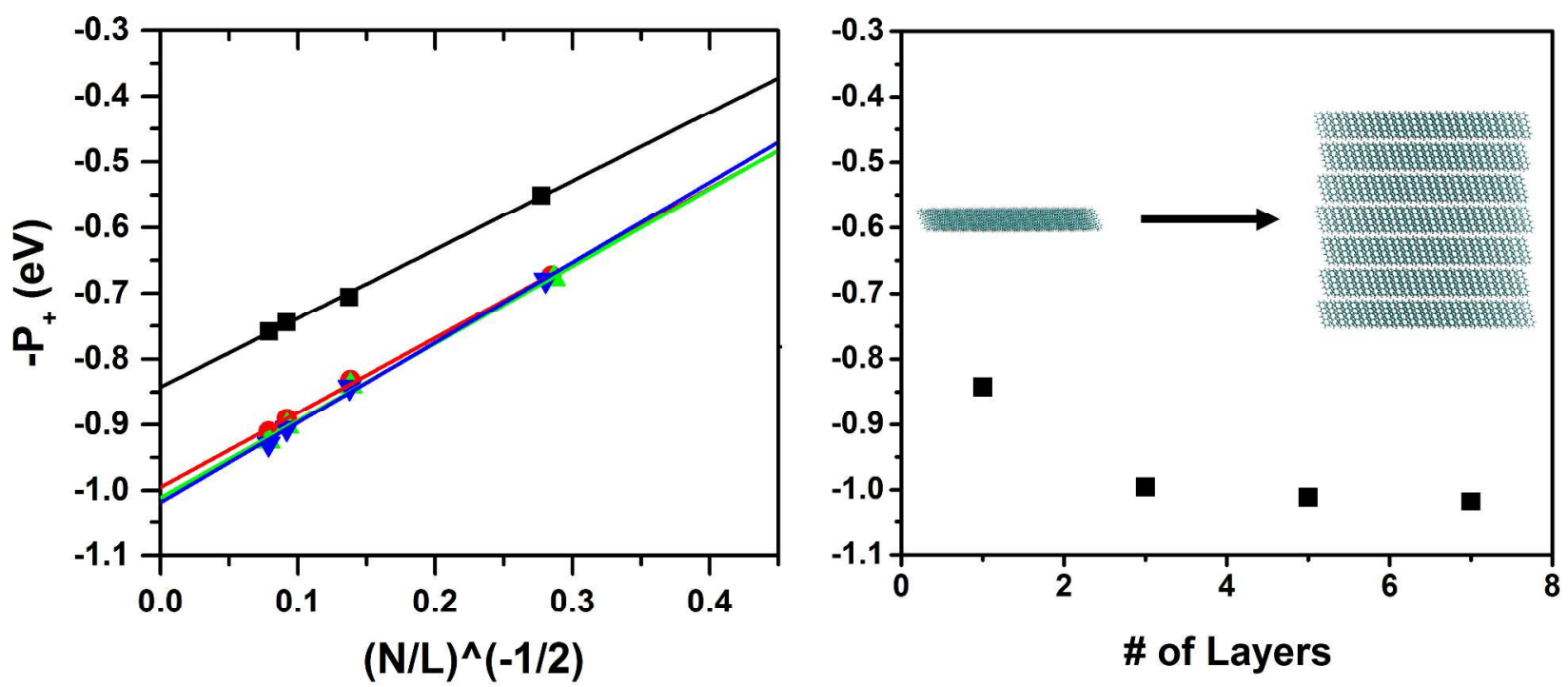

Figure 2. (Left) Polarization energy as a function of the average number of molecules per layer for cylinders of tetracene consisting of 1 layer (black; $\mathbf{a}), 3$ layers (red; •), 5 layers (green; $\triangle$ ), and 7 layers (blue; $\nabla$ ). (Right) Extrapolated polarization energies for cylinders of tetracene as the number of layers is increased.
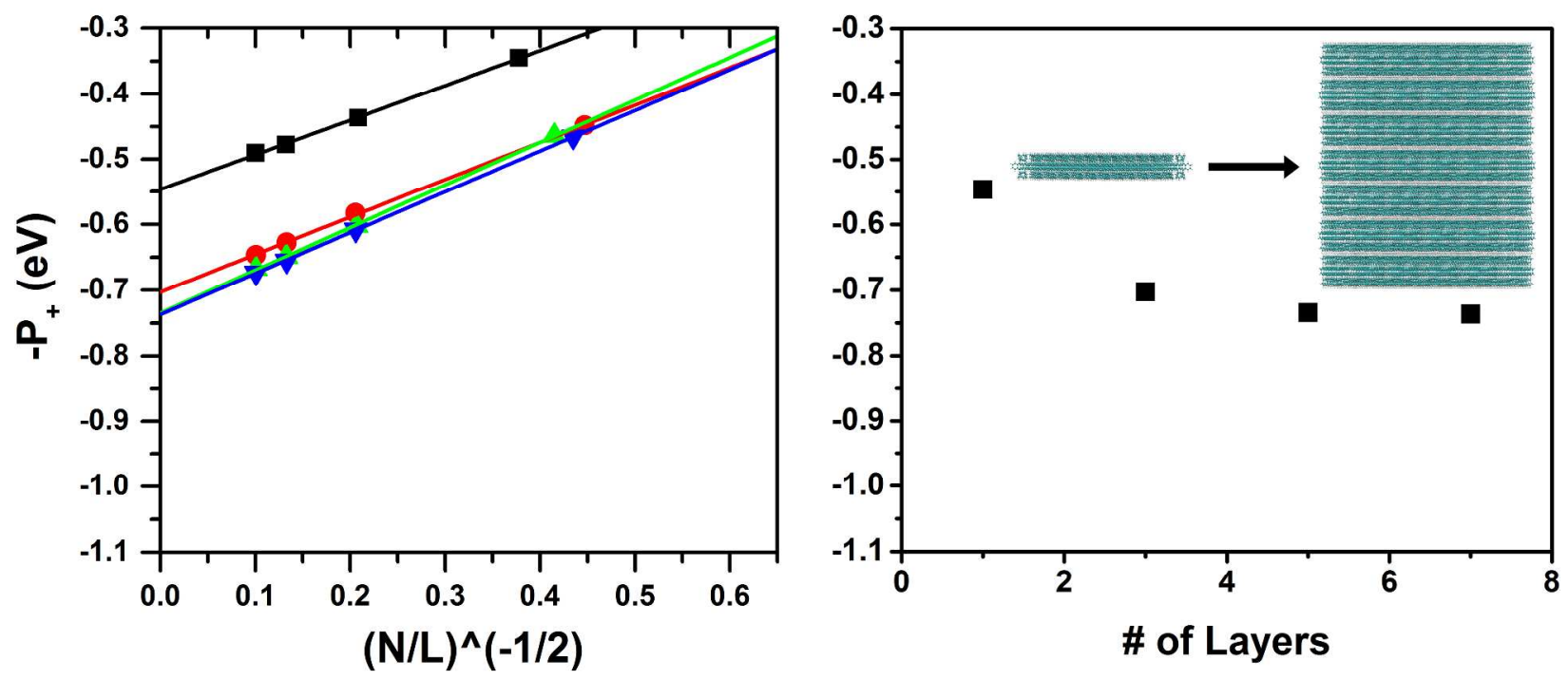

Figure 3. (Left) Polarization energy as a function of the average number of molecules per layer for cylinders of rubrene consisting of 1 layer (black; $\mathbf{a}), 3$ layers (red; •), 5 layers (green; $\triangle$ ), 
and 7 layers (blue; $\nabla$ ). (Right) Extrapolated polarization energies for cylinders of rubrene as the number of layers is increased.

\section{b) Polarization Energy as a Function of Interlayer and Intralayer Packing Densities}

Selective substitution of the phenyl rings of rubrene can allow for modulation of the molecular packing and thus modulation of the polarization energy. ${ }^{4}$ To better understand the variations in polarization energy, we investigated the effect of modifying the packing distances of rubrene and tetracene through expansion of the molecular crystal by as little as a 3\% reduction and up to a $75 \%$ reduction in packing density (Figure 4). First, we focus on expansions between layers, followed by expansion within molecular layers, and then, as an extreme limit, combined expansions within and between layers, which allows for a better understanding of how the polarization energy and intermolecular interactions evolve as a function of separation distance along the unit-cell axes.
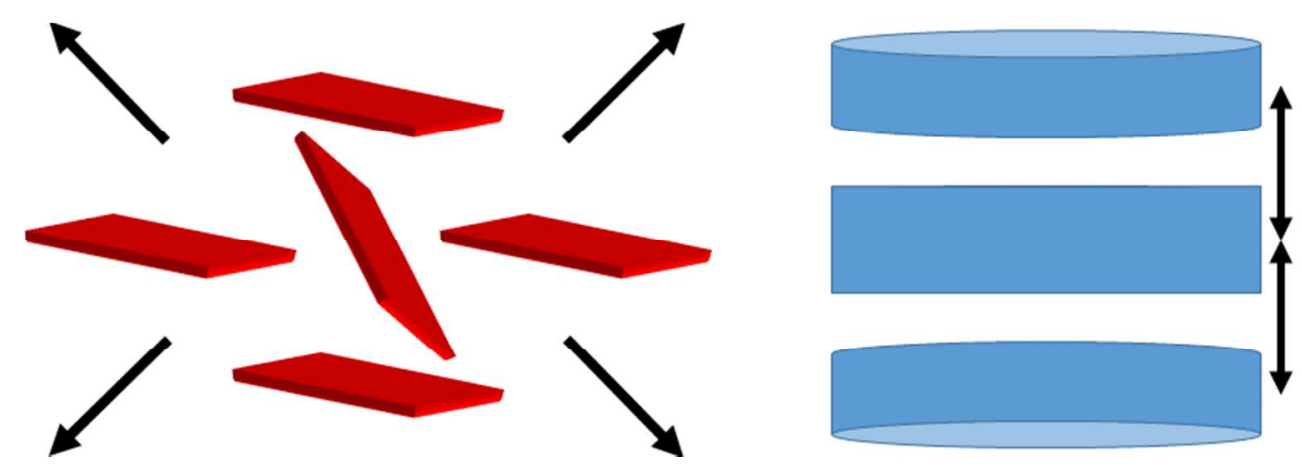

Figure 4. Illustrations of intralayer expansion (left) within the $a b$-plane and interlayer expansion (right) along the $c$-axis. 
As is the case with intermolecular electronic couplings (transfer integrals), the interactions between oligoacene molecules in the crystals can be expected to be weaker between layers than within layers. ${ }^{37-38}$ Accordingly, it is found that reducing the interlayer packing density results in a much smaller change than reducing the intralayer packing density for a given overall reduction in packing density. Figure 5 shows a decrease in the $P_{+}$polarization energy of about $0.1 \mathrm{eV}$ for both rubrene and tetracene as the distance between the layers is increased by $5 \AA$ from that determined crystallographically, which represents $27 \%$ and $15 \%$ reductions in the packing densities of tetracene and rubrene, respectively. As the separation increases to infinity, the polarization energy approaches that of the $2 D$-disk configurations (rubrene: $P_{+}=0.55 \mathrm{eV}$; tetracene: $P_{+}=0.84 \mathrm{eV}$ ). This limit is quickly reached as charge-quadrupole interactions have a $1 / r^{3}$ distance dependence while the charge-induced-dipole interactions can have stronger distance dependences determined by the nature of the induction source, be it a charge $\left(1 / r^{3}\right)$, a quadrupole $\left(1 / r^{5}\right)$, or an induced dipole (various distance dependences). 

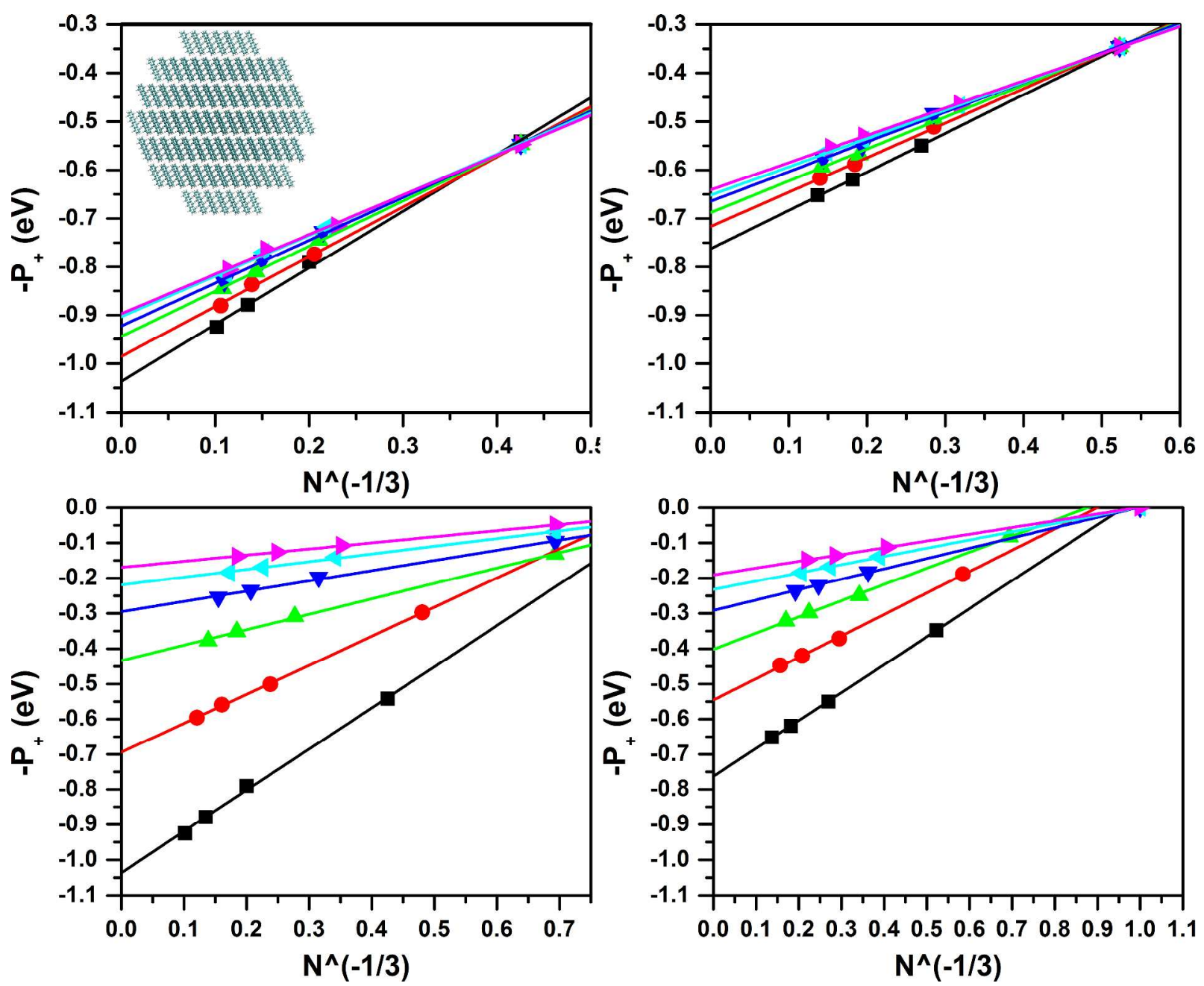

Figure 5. (Top) Extrapolated polarization energies of tetracene (left) and rubrene (right) as the interlayer separation distance is increased from the crystalline value (black; $\mathbf{a})$ by an additional 1 $\AA$ (red; •), $2 \AA$ (green; $\triangle$ ), $3 \AA$ (dark blue; $\nabla), 4 \AA$ (light blue; $<$ ), and $5 \AA$ (magenta; $>$ ). (Bottom) Extrapolated polarization energies of tetracene (left) and rubrene (right) as the intralayer separation distance is increased from the crystalline value to an additional $5 \AA$.

The polarization energy is significantly more sensitive to the intralayer packing density, leading to a much diminished polarization energy as the density is reduced. For rubrene, the polarization energy diminishes by $0.57 \mathrm{eV}$ (from $0.76 \mathrm{eV}$ to $0.19 \mathrm{eV}$ ) as the intralayer distance between molecules is increased by $5 \AA$ (a $56 \%$ reduction in packing density); Table S1 provides the relationships between a given increase along a unit-cell axis and the resulting density. The 
decrease in the polarization energy is even larger for tetracene, $0.87 \mathrm{eV}$ (from $1.04 \mathrm{eV}$ to $0.17 \mathrm{eV}$ with a $66 \%$ packing density reduction), a result of the larger dynamic component of the polarization energy and its strong distance dependence. For both systems, the polarization energy evolves not toward zero, but approaches that of a 1D chain stacked along the $c$-axis of the unit cell. Overall, these results are consistent with the fact that the intermolecular interactions for face-to-edge and face-to-face interactions (present within layers), where there are many interacting points (i.e., atoms), are significantly stronger than edge-to-edge interactions (present between layers). For example, comparing a tetracene cluster with $4 \AA$ of additional separation along the interlayer direction with a tetracene cluster with $1 \AA$ of additional separation along the intralayer direction, both with a packing density of $1.1 \mathrm{~g} / \mathrm{cm}^{3}$, results in polarization energies of $0.90 \mathrm{eV}$ and $0.69 \mathrm{eV}$, respectively.

Finally, we examine the extreme case of symmetric molecular expansion wherein both the intralayer and interlayer separations are concurrently increased at the same rate (Figure 6). In the range of expansions considered, we calculate a decrease in $P_{+}$of $0.92 \mathrm{eV}$ for tetracene and 0.65 $\mathrm{eV}$ for rubrene, corresponding to $75 \%$ and $63 \%$ packing density reductions, respectively. This expansion may be envisioned as beginning with a spherical cluster and then expanding the system to the point that it becomes a collection of non-interacting molecules. Thus, here, the polarization energy trends towards zero at the limit of infinite separation. 

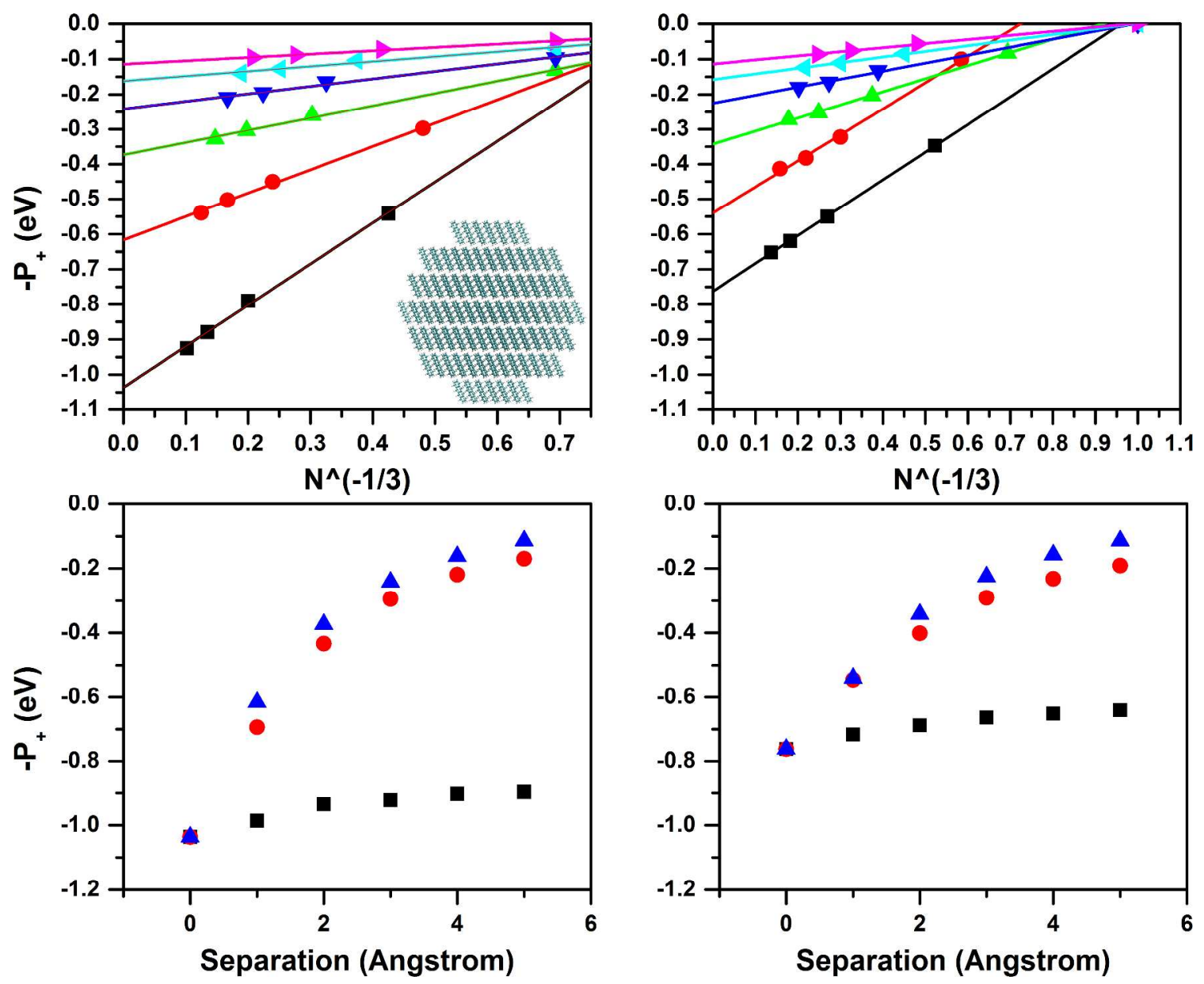

Figure 6. (Top) Extrapolated $P_{+}$polarization energies of tetracene (left) and rubrene (right) as both the interlayer and intralayer separation distances are increased from the crystalline value (black; 匹) by an additional $1 \AA$ (red; •), $2 \AA$ (green; $\triangle$ ), $3 \AA$ (dark blue; $\nabla$ ), $4 \AA$ (light blue; $<$ ), and $5 \AA$ (magenta; $>$ ). (Bottom) The combined polarization energies of tetracene (left) and rubrene (right) as a function of increased interlayer expansion (black; $\mathbf{a})$, intralayer expansion (red; •), and combined symmetric expansion (blue; $\boldsymbol{\Delta}$ ).

From a methodological standpoint, it should be noted that if the unit cell contains non-equivalent molecules, such as in the case of tetracene, then the same molecule must be used in both gasphase and solid-state calculations. If the same molecule is not used, then there will be a remainder of polarization energy at the limit of infinite expansion equivalent to the site energy difference between the two molecules (about $20 \mathrm{meV}$ for tetracene). We note that similar trends 
are observed for both hemispherical and cylindrical systems; the results are available in the Supporting Information.

\section{c) Bulk vs. Interfacial Polarization Energy}

Since the seminal work of Salaneck, ${ }^{21}$ there has been continued debate in the literature as to what extent the ionization energy, and in turn, the polarization energy at the surface of a material is different than that of the bulk. ${ }^{22-25}$ This difference is relevant, for instance, when discussing organic-vacuum field-effect transistors, where the charge moves along or near the vacuum interface, and thus can experience an energetic landscape different than the bulk. ${ }^{13}$ Changes in energetic landscape are also present at organic-organic and organic-inorganic interfaces common to organic electronic devices. Independent investigations by Tsiper and $\operatorname{Soos}^{24}$ and by Gorczak et $a l{ }^{25}$ confirm that indeed the environment at the organic-vacuum interface is different than the bulk; however, there is disagreement as to whether the change in the static and dynamic electrostatic interactions cancel each other out, as has been suggested by Gorczak et al. ${ }^{25}$ While these authors reported equivalent polarization energies in the bulk and at the surface of pentacene, Tsiper and Soos reported a difference of $0.23 \mathrm{eV}$, with the surface being less stabilized. ${ }^{24}$ Note that the treatment of Tsiper and Soos uses $\Delta P=P^{S}-P$, where $P=P_{+}+P_{-}$; $P^{S}=P_{+}^{S}+P_{-}^{S}$; and $P$ and $P^{S}$ denote the combined polarization energies due to a positive charge and negative charge in the bulk and at the surface, respectively.

To better assess the environment at the organic-vacuum interface, hemispherical clusters were used, where the organic material on one side of the layer in which the excess charge resides has been removed. The polarization energy due to a positive [negative] charge carrier at the tetracene [rubrene] surface is determined to be on the order of 0.07 [0.12] eV lower than the bulk value. 
When using the definition of Tsiper, we calculate $\Delta P$ to be $0.20 \mathrm{eV}$ and $0.17 \mathrm{eV}$ for tetracene and rubrene, respectively. The reduction in polarization energy calculated at the surface is lower than the value reported by Salaneck, $0.3 \mathrm{eV}$, but does clearly indicate that the electrostatics at the surface are distinct from the bulk. Simply determining the total difference in polarization energy between the surface and the bulk does not tell the entire story; as noted earlier, both static and dynamic interactions will change as a result of variations in the electrostatic environment.

To determine the impact of the changing electrostatic interactions, the polarization energy contributions of finite-size clusters (bulk) and hemispheres (interface) were examined (Table 1). With respect to the tetracene bulk, the static component of the $P_{+}$polarization energy at the very surface is more stabilizing by $0.06 \mathrm{eV}$ while the dynamic component (induced-dipole interactions) becomes less stabilizing by $0.13 \mathrm{eV}$. For rubrene, the static component becomes more stabilizing by $0.02 \mathrm{eV}$ and the dynamic component destabilizing by $0.09 \mathrm{eV}$. Both the increase in the static stabilization and decrease in dynamic stabilization result from a smaller number of neighboring molecules in the clusters; the static interactions become more stabilizing because of fewer destabilizing charge-quadrupole interactions, while the dynamic interactions become less stabilizing because of fewer polarizable points that always act to stabilize the charge. This is further illustrated when the molecules on both sides of the charged layer are removed, i.e., a situation corresponding to a two-dimensional disk. For tetracene, the stabilization due to the static contribution is almost doubled to $0.11 \mathrm{eV}$ while the destabilization due to the dynamic component increases to $0.26 \mathrm{eV}$. Similar changes in the static and dynamic contributions are observed for rubrene, stabilization by $0.04 \mathrm{eV}$ and destabilization by $0.20 \mathrm{eV}$, respectively. While both sides of the bulk material adjacent to the charged layer contribute equally to the polarization energy, they constitute a non-additive contribution due to the 
interaction of the static and induced moments between the two layers adjacent to the charged layer. Overall, an important result is that the removal of polarizable material, i.e., neighboring molecules, has a much larger impact on the polarization energy than the stabilization gained from fewer (destabilizing) static interactions, which ultimately lead to a smaller polarization energy at the surface than in the bulk, in line with the measurements of Salaneck. ${ }^{21}$

Table 1. Polarization energies due to an excess positive charge in rubrene and tetracene clusters, hemispherical organic-vacuum interfaces, and 2-dimensional disks. Static and dynamic interactions result from single-point calculations on finite-sized clusters of 4-nm radius. All energies are reported in $\mathrm{eV}$.

\begin{tabular}{l|ccc}
\hline Tetracene/Rubrene $(\mathrm{eV})$ & Spherical Cluster & Hemispherical Interface & 2D Disk \\
\hline $\mathrm{P}_{+}$ & $1.04 / 0.76$ & $0.89 / 0.63$ & $0.84 / 0.55$ \\
Static Interactions & $0.18 / 0.15$ & $0.24 / 0.17$ & $0.29 / 0.19$ \\
Dynamic Interactions & $0.74 / 0.50$ & $0.61 / 0.41$ & $0.48 / 0.30$ \\
\hline
\end{tabular}

In order to address more completely the results of UPS experiments, where electrons are expected to be ejected from the first few layers, we have expanded our analysis beyond examining just the very surface and the bulk. ${ }^{39-40}$ To do this, cylindrical systems of constant thickness were created where the excess charge is initially placed at the top-most layer and subsequently moved to deeper layers. The results are collected in Table 2. As the charge is moved from the surface of tetracene towards the bulk, the static polarization energy decreases by about $17 \%$ and the dynamic contribution increases by about $19 \%$. This results in an overall increase in the polarization energy by almost $10 \%$. Because of the different electrostatic 
interactions in rubrene, the decrease in static polarization energy is about $9 \%$ while the increase in the dynamic contribution is almost $21 \%$, again resulting in a larger polarization energy in the bulk than at the surface. Thus, while the changes in dynamic polarization energy for both rubrene and tetracene as a charge is moved from the surface to the bulk are similar, the different electrostatic interactions due to the variations in molecular packing significantly impact the static polarization energy of each system. Overall, these different interactions imply that in rubrene there is a much lower sensitivity of the static polarization energy with respect to charge depth.

When the charge is moved from the very surface to the layer below, there is a large increase in the polarization energy in both tetracene and rubrene, after which the change is only $1-2 \%$ as the charge moves more into the bulk. As discussed earlier, the layers directly neighboring the charge have the largest impact on the polarization energy with additional neighboring layers having comparatively little effect. The implications for (angle-resolved) UPS measurements are that only the very surface layer should be probed if the characteristics of the surface are desired, as penetration into any deeper layer quickly leads to results more representative of the bulk than of the surface. 
Table 2. Polarization energy of 4-nm radius cylindrical clusters of 7-layers as a cation is moved from the top-most interfacial layer to the middle layer that is representative of the bulk material. Static, dynamic, and static-plus-dynamic data represent finite-sized cylinders of 4-nm radius while $P_{+}$data are extrapolations. All energies are reported in $\mathrm{eV}$.

\begin{tabular}{l|cccc}
\hline $\begin{array}{l}\text { Tetracene/Rubrene } \\
(\mathrm{eV})\end{array}$ & Static $P_{+}$ & Dynamic $P_{+}$ & $S+D$ & $P_{+}$ \\
\hline Layer 1 (interface) & $0.23 / 0.16$ & $0.64 / 0.44$ & $0.87 / 0.59$ & $0.93 / 0.66$ \\
Layer 2 & $0.20 / 0.15$ & $0.73 / 0.51$ & $0.94 / 0.66$ & $1.01 / 0.73$ \\
Layer 3 & $0.19 / 0.15$ & $0.75 / 0.52$ & $0.94 / 0.67$ & $1.01 / 0.74$ \\
Layer 4 (bulk) & $0.19 / 0.15$ & $0.76 / 0.53$ & $0.95 / 0.67$ & $1.02 / 0.74$ \\
\hline
\end{tabular}

These results confirm that the environment of an excess charge at the surface of an organic material is different than that of an excess charge in the bulk, although not necessarily in the most intuitive manner. Furthermore, as shown for instance by Beljonne and co-workers, ${ }^{41}$ the presence of a second organic system will introduce strong interfacial electrostatic interactions that are expected to change further the energetic landscape and impact the behavior of an excess charge.

\section{Conclusions}

Using a combination of our model for polarization energy, simple multipole electrostatics, and various length scales, we have developed a description of the rubrene and tetracene organic molecular crystals which provides an understanding of how the intermolecular interactions change as a function of packing orientation and packing density. We have also shown how the 
energetic landscape at an organic-vacuum interface differs from that in the bulk, by breaking down the interactions into constituent static and dynamic components.

Beginning with the simplest dimer systems using multipole interactions, we have observed qualitative differences in the electrostatic interactions between tetracene and rubrene. In tetracene, there exist stabilizing face-to-edge interactions while in rubrene there occurs a competition between stabilizing face-to-edge interactions and strongly destabilizing face-to-face interactions. We then investigated the bulk materials as spherical clusters and cylinders, and evaluated the implications of reducing the packing density, by up to $75 \%$, on the bulk polarization energy.

The main conclusions are:

- The polarization energy due to a positive charge is $0.28 \mathrm{eV}$ larger in tetracene than rubrene, in good agreement with previous experimental reports.

- For both rubrene and tetracene, the polarization energy due to a positive charge is larger than that due to a negative charge.

- Since the rubrene backbone lies parallel to the $a b$-plane, the stabilization coming from induced dipoles is reduced compared to case of tetracene because of increased center-ofmass to center-of-mass distances.

- The polarization energy within a molecular layer does not saturate until about $4 \mathrm{~nm}$ from the charged molecule.

- The polarization energy due to interacting layers essentially saturates after the inclusion of one layer on either side of the charged layer, in contrast to previous studies that showed saturation after 5-10 molecular layers. ${ }^{24}$ 
- When the packing density is reduced, the intralayer packing density has a much larger influence on the polarization energy $(\sim 0.9 \mathrm{eV}$ reduction) than the interlayer packing density $(\sim 0.1 \mathrm{eV}$ reduction).

Thus, crystal engineering efforts similar to those of McGarry et $a l .^{4}$ could allow the polarization energy of a given material to be controlled by modifying the packing density along different crystallographic axes.

In the case of organic-vacuum interfaces, it is found that, as a result of the reduced number of neighboring sites, the static component of the polarization energy becomes more stabilizing while the dynamic component becomes much less stabilizing. Although the overall change in the polarization energy (about $0.1 \mathrm{eV}$ ) at the interface is relatively small compared to the total polarization energy (1.0 eV for tetracene), the changes in the individual static vs. dynamic components represent a much larger portion of the total and illustrate the significant modification in the electrostatic environment. Interestingly, any layer other than the very surface is more representative of the bulk environment than that of the surface.

Our results underline that, although the tetracene and rubrene molecules share the same backbone and their electronic properties and type of crystalline packing are similar, small changes in the electrostatic interactions among neighboring molecules can result in large changes in the polarization energy. The better understanding that this work has provided regarding the origin of the observed shifts in IEs of rubrene and tetracene at the surface as well as the nature of the intermolecular interactions influencing the polarization energy, will allow one to rationalize the variations in other materials that share similar structures but different packing morphologies. Overall, this work provides a general framework in the context of efforts to tune the polarization 
energy of organic electronic materials through molecular orientation and packing density and contributes to a much better insight of the nature of the electrostatic interactions at organicvacuum interfaces. 
Acknowledgements. This work has been supported by King Abdullah University of Science and Technology (KAUST), the KAUST Competitive Research Grant program, and the Office of Naval Research Global (Award N62909-15-1-2003). We acknowledge the IT Research Computing Team and Supercomputing Laboratory at KAUST for providing computational and storage resources. This work has also used the computing resources of the Garnet, Spirit, and Copper supercomputing systems through the DoD HPCMP. We thank Professor Dan Frisbie for most stimulating discussions and Professor Jay Ponder for invaluable assistance in troubleshooting the Tinker suite. C.R. thanks the University of Kentucky Vice President of Research for start-up funds.

Supporting Information Available: A table defining the relationships between unit-cell expansions and density as well as figures plotting the polarization energies of bulk tetracene and rubrene, the polarization energy of cylindrical organic-vacuum interfaces, the effect of reduced packing density in cylindrical, hemispherical, and organic-vacuum cylinder interfaces, extrapolated polarization energies as a function of depth of charge site, and the probing of the second geometry in the tetracene unit-cell, are available in the Supporting Information. This material is available free of charge via the Internet at http://pubs.acs.org. 


\section{References}

1. Braga, D.; Battaglini, N.; Yassar, A.; Horowitz, G.; Campione, M.; Sassella, A.; Borghesi, A., Bulk Electrical Properties of Rubrene Single Crystals: Measurements and Analysis. Phys. Rev. B 2008, 77 (11), 115205.

2. Hasegawa, T.; Takeya, J., Organic Field-Effect Transistors Using Single Crystals. Sci. Technol. Adv. Mater. 2009, 10 (2), 024314.

3. Troisi, A., Charge Transport in High Mobility Molecular Semiconductors: Classical Models and New Theories. Chem. Soc. Rev. 2011, 40 (5), 2347-2358.

4. McGarry, K. A.; Xie, W.; Sutton, C.; Risko, C.; Wu, Y.; Young, V. G.; Brédas, J.-L.; Frisbie, C. D.; Douglas, C. J., Rubrene-Based Single-Crystal Organic Semiconductors: Synthesis, Electronic Structure, and Charge-Transport Properties. Chem. Mater. 2013, 25 (11), 2254-2263.

5. Minder, N. A.; Ono, S.; Chen, Z.; Facchetti, A.; Morpurgo, A. F., Band-Like Electron Transport in Organic Transistors and Implication of the Molecular Structure for Performance Optimization. Adv. Mater. 2012, 24 (4), 503-508.

6. Anthony, J. E., Functionalized Acenes and Heteroacenes for Organic Electronics. Chem. Rev. 2006, 106 (12), 5028-5048.

7. Chien, C.-T.; Lin, C.-C.; Watanabe, M.; Lin, Y.-D.; Chao, T.-H.; Chiang, T.-c.; Huang, X.H.; Wen, Y.-S.; Tu, C.-H.; Sun, C.-H.; Chow, T. J., Tetracene-Based Field-Effect Transistors Using Solution Processes. J. Mater. Chem. 2012, 22 (26), 13070-13075.

8. Eilmes, A., A Theoretical Study of Small Tetracene Clusters: Stability and Charge Stabilization Energies. Comput. Theor. Chem. 2012, 982 (0), 25-29.

9. Pope, M.; Burgos, J.; Giachino, J., Charge-Transfer Exciton State and Energy Levels in Tetracene Crystal. J. Chem. Phys. 1965, 43 (9), 3367-3371.

10. Sakamoto, Y.; Suzuki, T.; Kobayashi, M.; Yuan, G.; Inoue, Y.; Tokito, S., Perfluoropentacene and Perfluorotetracene: Syntheses, Crystal Structures, and FET Characteristics. Mol. Cryst. Liq. Cryst. 2006, 444 (1), 225-232.

11. Xia, Y.; Kalihari, V.; Frisbie, C. D.; Oh, N. K.; Rogers, J. A., Tetracene Air-Gap SingleCrystal Field-Effect Transistors. Appl. Phys. Lett. 2007, 90 (16), 162106.

12. Jurchescu, O. D.; Baas, J.; Palstra, T. T. M., Effect of Impurities on the Mobility of Single Crystal Pentacene. Appl. Phys. Lett. 2004, 84 (16), 3061-3063.

13. Coropceanu, V.; Cornil, J.; Silva, D. A. d.; Olivier, Y.; Silbey, R.; Bredas, J. L., Charge Transport in Organic Semiconductors. Chem. Rev. 2007, 107 (4), 926-952.

14. Silinsh, E. A., Organic Molecular Crystals: Their Electronic States. Springer: New York, 1980 .

15. Silinsh, E. A.; Capek, V., Organic Molecular Crystals: Interaction, Localization, and Transport Phenomena. AIP: New York, 1994.

16. Lyons, L., Ionized States of Molecular Crystals. Aust. J. Chem. 1957, 10 (3), 365-367.

17. Sato, N.; Seki, K.; Inokuchi, H., Polarization Energies of Organic Solids Determined by Ultraviolet Photoelectron Spectroscopy. J. Chem. Soc., Faraday Trans. 2 1981, 77, 1621-1633.

18. Sato, N.; Inokuchi, H.; Silinsh, E. A., Reevaluation of Electronic Polarization Energies in Organic Molecular Crystals. Chem. Phys. 1987, 115 (2), 269-277.

19. Ryno, S. M.; Risko, C.; Brédas, J.-L., Impact of Molecular Packing on Electronic Polarization in Organic Crystals: The Case of Pentacene vs TIPS-Pentacene. J. Am. Chem. Soc. 2014, 136 (17), 6421-6427. 
20. Ryno, S. M.; Lee, S. R.; Sears, J.; Risko, C.; Bredas, J. L., Electronic Polarization Effects upon Charge Injection in Oligoacene Molecular Crystals: Description via a Polarizable Force Field. J. Phys. Chem. C 2013, 117, 13853-13860.

21. Salaneck, W., Intermolecular Relaxation Energies in Anthracene. Phys. Rev. Lett. 1978, 40 (1), 60-63.

22. Casu, M. B.; Zou, Y.; Kera, S.; Batchelor, D.; Schmidt, T.; Umbach, U., Investigation of Polarization Effects in Organic Thin Films by Surface Core-Level Shifts. Phys. Rev. B 2007, 76, 193311.

23. Yoshida, H.; Sato, N., A Precise Analysis of the Core-Level Energy Difference between the Surface and Bulk Region of Organic Semiconductor Thin Films. J. Phys. Chem. C 2012, 116 (18), 10033-10038.

24. Tsiper, E. V.; Soos, Z. G., Electronic Polarization in Pentacene Crystals and Thin Films. Phys. Rev. B 2003, 68 (8), 085301.

25. Gorczak, N.; Swart, M.; Grozema, F. C., Energetics of Charges in Organic Semiconductors and at Organic Donor-Acceptor Interfaces. J. Mater. Chem. C 2014, 2 (17), 3467-3475.

26. Holmes, D.; Kumaraswamy, S.; Matzger, A. J.; Vollhardt, K. P. C., On the Nature of Nonplanarity in the [N]Phenylenes. Chem. Eur. J. 1999, 5, 3399-3412.

27. Jurchescu, O. D.; Meetsma, A.; Palstra, T. T. M., Low-Temperature Structure of Rubrene Single Crystals Grown by Vapor Transport. Acta Crystallogr., Sect. B: Struct. Sci. 2006, 62 (2), 330-334.

28. Allen, F. H., The Cambridge Structural Database: A Quarter Million Crystal Structures and Rising. Acta Crystallogr. 2002, B58, 380-388.

29. Fletcher, D. A.; McMeeking, R. F.; Parkin, D., The United Kingdom Chemical Database Service. J. Chem. Inf. Comput. Sci. 1996, 36, 746-749.

30. Densities were determined using the unit cell parameters and atoms per unit cell.

31. Ren, P.; Ponder, J. W., Polarizable Atomic Multipole Water Model for Molecular Mechanics Simulation. J. Phys. Chem. B 2003, 107 (24), 5933-5947.

32. Ponder, J. W.; Wu, C.; Ren, P.; Pande, V. S.; Chodera, J. D.; Schnieders, M. J.; Haque, I.; Mobley, D. L.; Lambrecht, D. S.; DiStasio, R. A.; Head-Gordon, M.; Clark, G. N. I.; Johnson, M. E.; Head-Gordon, T., Current Status of the AMOEBA Polarizable Force Field. J. Phys. Chem. B 2010, 114 (8), 2549-2564.

33. Stone, A. J., Distributed Multipole Analysis, or How to Describe a Molecular Charge Distribution. Chem. Phys. Lett. 1981, 83 (2), 233-239.

34. Frisch, M. J.; Trucks, G. W.; Schlegel, H. B.; Scuseria, G. E.; Robb, M. A.; Cheeseman, J. R.; Scalmani, G.; Barone, V.; Mennucci, B.; Petersson, G. A.; Nakatsuji, H.; Caricato, M.; Li, X.; Hratchian, H. P.; Izmaylov, A. F.; Bloino, J.; Zheng, G.; Sonnenberg, J. L.; Hada, M.; Ehara, M.; Toyota, K.; Fukuda, R.; Hasegawa, J.; Ishida, M.; Nakajima, T.; Honda, Y.; Kitao, O.; Nakai, H.; Vreven, T.; Montgomery, J. A.; Peralta, J. E.; Ogliaro, F.; Bearpark, M.; Heyd, J. J.; Brothers, E.; Kudin, K. N.; Staroverov, V. N.; Kobayashi, R.; Normand, J.; Raghavachari, K.; Rendell, A.; Burant, J. C.; Iyengar, S. S.; Tomasi, J.; Cossi, M.; Rega, N.; Millam, J. M.; Klene, M.; Knox, J. E.; Cross, J. B.; Bakken, V.; Adamo, C.; Jaramillo, J.; Gomperts, R.; Stratmann, R. E.; Yazyev, O.; Austin, A. J.; Cammi, R.; Pomelli, C.; Ochterski, J. W.; Martin, R. L.; Morokuma, K.; Zakrzewski, V. G.; Voth, G. A.; Salvador, P.; Dannenberg, J. J.; Dapprich, S.; Daniels, A. D.; Farkas; Foresman, J. B.; Ortiz, J. V.; Cioslowski, J.; Fox, D. J., Gaussian 09, Revision B.01. Wallingford CT, 2009. 
35. Berry, R. S.; Jortner, J.; Mackie, J. C.; Pysh, E. S.; Rice, S. A., Search for a Charge-Transfer State in Crystalline Anthracene. J. Chem. Phys. 1965, 42 (5), 1535-1540.

36. Stone, A. J., The Theory of Intermolecular Forces. Clarendon Press: Oxford, 1996.

37. Delgado, M. C. R.; Pigg, K. R.; da Silva Filho, D. A.; Gruhn, N. E.; Sakamoto, Y.; Suzuki, T.; Osuna, R. M.; Casado, J.; Hernández, V.; Navarrete, J. T. L.; Martinelli, N. G.; Cornil, J.; Sánchez-Carrera, R. S.; Coropceanu, V.; Brédas, J.-L., Impact of Perfluorination on the ChargeTransport Parameters of Oligoacene Crystals. J. Am. Chem. Soc. 2009, 131 (4), 1502-1512.

38. Salman, S.; Delgado, M. C. R.; Coropceanu, V.; Brédas, J.-L., Electronic Structure and Charge-Transport Parameters of Functionalized Tetracene Crystals: Impact of Partial Fluorination and Alkyl or Alkoxy Derivatization. Chem. Mater. 2009, 21 (15), 3593-3601.

39. Seah, M. P.; Dench, W. A., Quantitative Electron Spectroscopy of Surfaces: A Standard Data Base for Electron Inelastic Mean Free Paths in Solids. Surf. Interface Anal. 1979, 1 (1), 211 .

40. Tanuma, S.; Powell, C. J.; Penn, D. R., Calculations of Electron Inelastic Mean Free Paths. V. Data for 14 Organic Compounds Over the 50-2000 eV Range. Surf. Interface Anal. 1994, 21 (3), 165-176.

41. Linares, M.; Beljonne, D.; Cornil, J. r. m.; Lancaster, K.; Brédas, J.-L.; Verlaak, S.; Mityashin, A.; Heremans, P.; Fuchs, A.; Lennartz, C.; Idé, J.; Méreau, R. 1.; Aurel, P.; Ducasse, L.; Castet, F. d. r., On the Interface Dipole at the Pentacene-Fullerene Heterojunction: A Theoretical Study. J. Phys. Chem. C 2010, 114 (7), 3215-3224. 
Table of Contents Figure
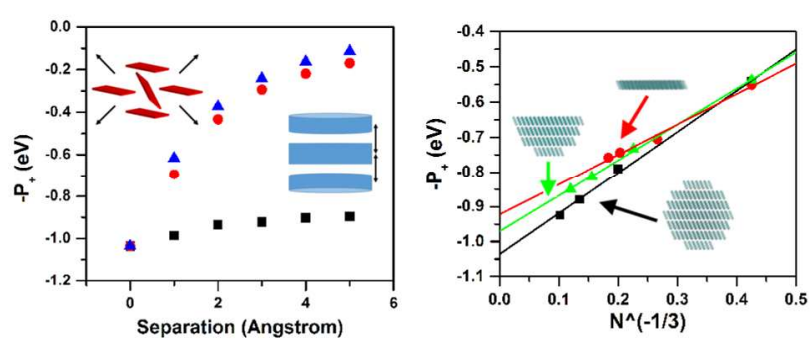

16

17

18

19

20

21

22

23

24

25

26

27

28

29

30

31

32

33

34

35

36

37

38

39

40

41

42

43

44

45

46

47

48

49

50

51

52

53

54

55

56

57

58

59

60 